\title{
Case study from the Garvan Institute of Medical Research: long-term survival of a patient with metastatic breast cancer and hypercalcaemia
}

\author{
C. McNeila, J. Grygiela,b \\ ${ }^{a}$ Garvan Institute of Medical Research, ${ }^{b}$ Department of Medical Oncology, St. Vincent's Hospital, \\ Darlinghurst, NSW, Australia.
}

\section{Presentation and diagnosis}

In June 1998, a 50-year-old woman presented to hospital with vomiting, dehydration, serum calcium of $4.7 \mathrm{mmol} / \mathrm{l}$ and deranged liver function tests. Prior to her presentation she had been unwell for 6 weeks with polyuria and anorexia.

She was rehydrated and her calcium was corrected with intravenous pamidronate. Investigations revealed undetectable parathyroid hormone (PTH) and multiple liver lesions, the largest measuring $6 \mathrm{~cm}$. Biopsy of the liver lesions confirmed metastatic adenocarcinoma (? site of origin). There was insufficient tissue to assess for hormone receptors. A breast mass measuring $3 \mathrm{~cm}$ was identified in the left breast in retrospect, consistent with a diagnosis of metastatic breast cancer.

\section{Treatment and outcome}

In July 1998 anti-neoplastic treatment was commenced with two cycles of epirubicin/cyclophosphamide $\left(60 \mathrm{mg} / \mathrm{m}^{2}+600 \mathrm{mg} / \mathrm{m}^{2} \mathrm{q} 3\right.$ weekly) that was then changed to epirubicin/docetaxel $\left(60 \mathrm{mg} / \mathrm{m}^{2}+\right.$ $100 \mathrm{mg} / \mathrm{m}^{2}$ ) because of disease progression. This was continued for a further 10 cycles with a resultant progressive partial response. (Cardiac toxicity screening was undertaken with echocardiography and no significant decrease in ventricular function was identified.) Subsequently she was maintained on tamoxifen

Correspondence to: Dr C. McNeil, MBBS, FRACP, Garvan Institute of Medical Research, St. Vincent's Hospital, Darlinghurst, NSW 2010, Australia. E-mail: c.mcneil@garvan.org.au; Tel: +61 29295 8322; Fax: +61 292958321

Received 04/08/05

Accepted 04/08/05

BCO/414/2005/CS
$20 \mathrm{mg}$ daily with continuing response on computed tomography (CT) scan. Her disease remained stable from February 2000 to October 2002, at which time she developed progressive liver metastases and therapy was changed to anastrazole $1 \mathrm{mg}$ daily. This has been maintained until the present time. Slowly progressive bone disease was noted on bone scan in January 2004 and she has recently been found to have progressive liver metastases. Monthly pamidronate $90 \mathrm{mg}$ was maintained throughout her therapy from diagnosis (changed to zoledronate $4 \mathrm{mg}$ in April 2003) and continues to the present time. Her calcium level has remained normal throughout.

In summary this woman has remained well on a combination of chemotherapy and anti-oestrogen therapy for 7 years after initially presenting with profound hypercalcaemia and advanced metastatic breast cancer.

\section{Comments}

Metastatic disease comprises approximately $6-10 \%$ of new breast cancer presentations. In a recent study of women presenting with metastatic breast cancer, 343 women diagnosed between 1987 and 1993 (Group 1) and 381 women diagnosed between 1994 and 2001 (Group 2) were evaluated [1]. In these matched cohorts, the 5-year survival was $11 \%$ in Group 1 and 28\% in Group 2. The use of new treatments such as aromatase inhibitors and taxanes was significantly higher in Group 2 (58\% vs. 10\%). The difference in survival was most apparent in the hormone receptor positive subgroup where the 5-year survival was $14 \%$ in Group 1 and $38 \%$ in Group 2 $(P<0.001)$. These data are consistent with analyses demonstrating that outcomes for women with 
metastatic breast cancer are improved by taxanecontaining regimens, combination chemotherapy, and aromatase inhibition [2-5].

It is therefore clear that prolonged survival is achievable in the era of new endocrine therapies and taxanebased chemotherapy (both of which our patient received). It is notable that the patient described above remained well and with stable disease for over 4 years on anti-hormonal therapies and bisphosphonates alone.

What is perhaps more striking is her prolonged survival after initially presenting with severe hypercalcaemia. In general, the median survival of breast cancer patients with hypercalcaemia is poor at around 6 months. The survival is shorter still in the severely hypercalcaemic cohort. The survival from breast cancer related hypercalcaemia in the pre- and postbisphosphonate era has been evaluated in 244 patients in a study from Denmark [6]. Median survival for the severely hypercalaemic subgroup of patients was 1.4 months in the period prior to bisphosphonate therapy (1986-1989) and 2.2 months after the introduction of bisphosphonate therapy (1993-1996) in this study, which included patients in both the early and late phases of their cancer treatment. While it is well known that bisphosphonates have quality of life benefits relating to reduced skeletal-related morbidity, there has been no demonstrated survival benefit [7], and therefore, it is unlikely that the use of pamidronate and zoledronate contributed significantly to our patient's prolonged survival.

Thus the survival and excellent performance status of our patient is remarkable given her initial presentation and highlights the fact that prolonged survival is achievable with advanced breast cancer using new endocrine and chemotherapeutic agents. The promise of molecular biology poses the future challenge of identifying those patients who will gain maximal benefit from such therapies.

\section{Acknowledgments}

Dr McNeil is supported by National Health and Medical Research Council and New South Wales Cancer Institute Scholarships.

\section{References}

1. Andre $F$, Slimane $K$, Bachelot $T$, et al. Breast cancer with synchronous metastases: trends in survival during a 14-year period. J Clin Oncol 2004; 22: 3302-3308.

2. Bonneterre J, Buzdar A, Nabholtz JM, et al. Anastrazole is superior to tamoxifen as first-line therapy in hormone receptor positive advanced breast carcinoma. Cancer 2001; 92: 2247-2258.

3. Buzdar A, Jonat W, Howell A, et al. Anastrazole, a potent and selective aromatase inhibitor, versus megestrol acetate in post menopausal women with advanced breast cancer: results of overview analysis of two phase III trials. Arimidex Study Group. J Clin Oncol 1996; 14: 2000-2011.

4. Carrick S, Parker S, Wilcken N, et al. Single agent versus combination chemotherapy for metastatic breast cancer. The Cochrane Database of Systematic Reviews 2005; Issue 2. Art. No.: CD003372.pub2. DOI: 10.1002/ 14651858.CD003372.pub2.

5. Ghersi D, Wilcken N, Simes J, Donoghue E. Taxane containing regimens for metastatic breast cancer. The Cochrane Database of Systematic Reviews 2005; Issue 2. Art. No.: CD003366.pub2. DOI: 10.1002/14651858. CD003366.pub2.

6. Kristensen B, Ejlertsen B, Mouridsen HT, et al. Survival in breast cancer patients after the first episode of hypercalcaemia. J Int Med 1998; 244: 189-198.

7. Pavlakis N, Stockler M. Bisphosphonates for breast cancer. The Cochrane Database of Systematic Reviews 2002; Issue 1. Art. No.: CD003474. DOI: 10.1002/ 14651858.CD003474. 